\title{
Are corporates' target leverage ratios time-dependent?
}

\author{
C.H. Hui ${ }^{a, *}$, C.F. Lo ${ }^{\text {b, }}{ }^{\text {, M.X. Huang }}{ }^{\text {b, } 1}$ \\ ${ }^{a}$ Research Department, Hong Kong Monetary Authority, 55th Floor, Two International Financial Centre, \\ 8 Finance Street, Central, Hong Kong SAR, China \\ ${ }^{\mathrm{b}}$ Department of Physics and Institute of Theoretical Physics, The Chinese University of Hong Kong, Shatin, New \\ Territories, Hong Kong SAR, China
}

Received 3 October 2004; accepted 4 January 2005

Available online 4 November 2005

\begin{abstract}
This paper extends the stationary-leverage-ratio model to incorporate a time-dependent target leverage ratio. The theoretical hypothesis of the existence of a time-dependent target leverage ratio reflects the movement of a firm's initial target ratio toward a long-term target ratio over time. Using some simple scenarios about the time-dependence of the target leverage ratio, the numerical results show that the incorporation of the hypothesis into the stationary-leverage-ratio model is capable of producing term structures of probabilities of default that are consistent with some empirical findings. The results provide some evidences to support the hypothesis.
\end{abstract}

(C) 2005 Elsevier Inc. All rights reserved.

JEL classification: G21; G28; G13

Keywords: Credit risk models; Credit ratings; Default probability

\section{Introduction}

Black and Scholes (1973) and Merton (1974) have been the pioneers in the pricing of corporate bonds using a contingent-claims framework. ${ }^{2}$ In Black-Scholes-Merton's structural framework, a firm's market value of total assets is observable in principle. Furthermore the

\footnotetext{
* Corresponding author. Tel.: +852 2878 1485; fax: +852 28781899.

E-mail addresses: cho-hoi_hui@hkma.gov.hk (C.H. Hui), cflo@sun1.phy.cuhk.edu.hk (C.F. Lo).

${ }^{1}$ Tel.: +852 2609 6362; fax: +852 26035204 .

2 The second approach is the reduced-form models in which default time is a stopping time of some given hazard rate process and the payoff upon default is specified exogenously. This approach has been considered by Madan and Unal (1993), Jarrow, Lando, and Turnbull (1994), Jarrow and Turnbull (1995), and Duffie and Singleton (1997).
} 
capital structure is explicitly considered and default happens if the total asset value is lower than the value of liabilities. Default risk is therefore equivalent to a European put option on the firm's asset value.

To cope with the possibility of early default prior to maturity in the structural model, Black and Cox (1976) assume a default-triggering level for the firm's asset value whereby default can occur at any time. This trigger level is introduced by considering a safety covenant that protects bondholders. Longstaff and Schwartz (1995) extend Black and Cox's model to allow interest rates to follow the Ornstein-Uhlenbeck process. Default occurs when the firm's asset value is below a constant default barrier. The constant default barrier in the Longstaff and Schwartz model corresponds to the total amount of debts issued by the firm, that is kept constant over time. The model therefore predicts that the expected liability-to-asset ratio (i.e. leverage ratio) will decline exponentially over time. The decline in the expected leverage ratio is however not supported by empirical observations.

Briys and de Varenne (1997) develop a corporate bond pricing model which is characterised by a default barrier following the dynamics of the risk-free interest rate. As a result, the default barrier is assumed to grow together with the firm value over time and the expected level of leverage is therefore kept constant. This assumption may be appropriate in case the firm is neither willing nor able to adjust its expected level of leverage over time. To model a more flexible default barrier, Hui, Lo, and Tsang (2003) consider a dynamic default barrier which incorporates scenarios of high default risk at time close to bond maturity and the characteristics of both the Longstaff and Schwartz model and the Briys and de Varenne model.

Empirical findings, including Marsh (1982), Jalivand and Harris (1984), Auerbach (1985) and Opler and Titman (1995), document that companies tend to gradually adjust their capital structures toward a target level of leverage. This means that a firm adjusts its outstanding debts in response to changes in its firm value in order to achieve a target level of leverage. These findings call for the stationary-leverage-ratio model for pricing corporate bonds, which has been studied by Collin-Dufresne and Goldstein (2001). Their structural model considers a meanreverting liability that is tied to the interest rate dynamics. The leverage ratio is defined as a ratio of the liability to the asset value of a firm. The model parameters in the Collin-Dufresne and Goldstein's model (hereafter referred to as the CG model) are all constant. These assumptions make the leverage ratio approach towards a constant target leverage ratio over time. CollinDufresne and Goldstein observe empirically that the long-term target ratio is close to the average leverage ratio of BBB-rated firms. They conclude that accounting for a bond issuer's ability to control its level of outstanding debt in the model has a significant impact on credit spread predictions. It helps reconcile some predictions of credit spreads with empirical observations. These include credit spreads that are larger for low-leverage firms and less sensitive to changes in firm value, and upward sloping term structures of credit spreads of speculative-grade bonds.

As the long-term target leverage ratio may be very different from the short-term ratio, we expect the target leverage ratio should vary with time. The objective of this paper is to propose a theoretical hypothesis of the existence of a time-dependent target leverage ratio. To check the validity of this theoretical hypothesis, we extend the model through incorporating a timedependent target leverage ratio into the model (hereafter referred to as the time-dependent model). In the time-dependent model the firm's liability is assumed to be governed by a meanreverting stochastic process whilst the firm value follows a simple lognormal process. The two stochastic variables of the firm value and the liability are correlated. By incorporating timedependent model parameters in the model, the target leverage ratio is thus time-dependent. The time-dependent target leverage ratio can therefore reflect the movement of a firm's initial target 
leverage ratio toward a long-term target ratio over time which is the average leverage ratio of BBB-rated firms. When the volatility of the liability is set equal to zero and the model parameters are constant, the time-dependent model converges to the CG model.

The time-dependent model assumes that default occurs when a firm's leverage ratio increases above a predefined default-triggering level. The dynamics of the short-term interest rate in the model is assumed to follow the Ornstein-Uhlenbeck process, i.e. the Vasicek model (Vasicek, 1977). It is correlated with the firm value and the firm liability. A closed-form solution is derived to compute model probabilities of default (PDs) based on the time-dependent model. Using market data for corporates with different credit ratings, the PDs generated from the model show that the use of some simple, yet non-trivial, scenarios about the time-dependence of the target leverage ratio (where the initial target ratio decreases gradually toward the average leverage ratio of BBB-rated firms over time) is capable of producing term structures of PDs that are consistent with the empirically observed cumulative default rates of different ratings reported by Standard and Poor's (S\&P's) (2002). On the other hand, the model PDs generated from the CG model are substantially lower than the empirical default rates. The numerical results provide some evidences to support the hypothesis of the existence of a time-dependent target leverage ratio.

The scheme of this paper is as follows. In the following section we discuss the timedependent model and derive the closed-form solution for estimating model PDs. Numerical results of PDs calculated from the model based on market data are then compared with the default rates reported by S\&P's and model PDs generated from the CG and Merton models. The effects of the correlation among the stochastic variables in the time-dependent model on PDs are also studied. At the end we will summarise our investigation.

\section{Analytical model of PD}

A continuous-time framework is used to value PD of a firm in the model. The firm value $V$ is assumed to follow a lognormal diffusion process. The firm liability $Q$ is governed by a meanreverting lognormal diffusion process. ${ }^{3}$ The dynamics of the risk-free interest rate $r$ is drawn from the term structure model of Vasicek (1977), i.e. the Ornstein-Uhlenbeck process. ${ }^{4}$ Their continuous stochastic movements are modelled by the following stochastic differential equations:

$$
\begin{aligned}
& \frac{\mathrm{d} V}{V}=\mu_{V}(t) \mathrm{d} t+\sigma_{V}(t) \mathrm{d} Z_{V} \\
& \frac{\mathrm{d} Q}{Q}=\left\{\mu_{Q}(t)+\kappa_{Q}(t)(\ln V-\ln Q)\right\} \mathrm{d} t+\sigma_{Q}(t) \mathrm{d} Z_{Q} \\
& \mathrm{~d} r=\kappa_{r}(t)\left[\theta_{r}(t)-r\right] \mathrm{d} t+\sigma_{r}(t) \mathrm{d} Z_{r}
\end{aligned}
$$

where $\sigma_{Q}(t)$ and $\sigma_{V}(t)$ are the respective volatility values, $\mu_{Q}(t)$ and $\mu_{V}(t)$ are the respective drift rates, and the firm liability $Q$ is mean-reverting at speed $\kappa_{Q}(t)$. When $\ln Q$ is less than $\ln V$, the firm acts to increase $\ln Q$, and vice-versa. This means that the firm tends to issue debt when its leverage ratio falls below a target and reduces its liability when its leverage ratio is above the target. Future changes in the liability structures of the firm give uncertainty to the value of the

\footnotetext{
${ }^{3} Q$ can be the market value of the total debts or the present value of the face value of the liabilities.

4 Although this assumed process is consistent with many of the observed properties of interest rates, it can allow negative interest rates. However, this assumption may still be justifiable in the context of the valuation because given that the current value of interest rate and the mean-level are both positive, the dynamics always imply positive expected future interest rate.
} 
liability. There is no other explicit relationship assumed between the firm value and the way it will impact the value of the liability. The interest rate uncertainty is driven by a Vasicek representation with the instantaneous volatility $\sigma_{r}(t)$. The short-term interest rate $r$ is meanreverting to long-run mean $\theta_{r}(t)$ at speed $\kappa_{r}(t)$. All model parameters are explicitly timedependent. The Wiener processes $\mathrm{d} Z_{Q}, \mathrm{~d} Z_{V}$ and $\mathrm{d} Z_{r}$ are correlated with

$$
\begin{aligned}
& \mathrm{d} Z_{V} \mathrm{~d} Z_{Q}=\rho_{V Q}(t) \mathrm{d} t \\
& \mathrm{~d} Z_{V} \mathrm{~d} Z_{r}=\rho_{V r}(t) \mathrm{d} t \\
& \mathrm{~d} Z_{Q} \mathrm{~d} Z_{r}=\rho_{Q r}(t) \mathrm{d} t .
\end{aligned}
$$

We define $R \equiv Q / V$ to be the leverage ratio and apply the Ito's lemma to derive the partial differential equation governing a corporate discount bond value $P(R, r, t)$ based on the model as follows: (see the Appendix)

$$
\begin{aligned}
\frac{\partial P(R, r, t)}{\partial t}= & \frac{1}{2} \sigma_{R}^{2}(t) R^{2} \frac{\partial^{2} P}{\partial R^{2}}+\frac{1}{2} \sigma_{r}^{2}(t) \frac{\partial^{2} P}{\partial r^{2}}+\rho_{R r}(t) \sigma_{R}(t) \sigma_{r}(t) R \frac{\partial^{2} P}{\partial R \partial r} \\
& +\kappa_{Q}(t)\left[\ln \theta_{R}(t)-\ln R\right] R \frac{\partial P}{\partial R}+\kappa_{r}(t)\left[\theta_{r}(t)-r\right] \frac{\partial P}{\partial r}-r P
\end{aligned}
$$

where $t$ is the time-to-maturity,

$$
\begin{aligned}
\sigma_{R}(t) & \equiv \sqrt{\sigma_{Q}^{2}(t)-2 \rho_{Q V}(t) \sigma_{Q}(t) \sigma_{V}(t)+\sigma_{V}^{2}(t)}, \\
\rho_{R r}(t) & \equiv \frac{\rho_{Q r}(t) \sigma_{Q}(t)-\rho_{V r}(t) \sigma_{V}(t)}{\sigma_{R}(t)}, \\
\theta_{R}(t) & \equiv \exp \left\{\left[\sigma_{V}^{2}(t)-\rho_{Q V}(t) \sigma_{Q}(t) \sigma_{V}(t)-\delta(t)\right] / \kappa_{Q}(t)\right\}, \\
\delta(t) & \equiv \lambda_{Q}(t) \sigma_{Q}(t)-\lambda_{V}(t) \sigma_{V}(t) .
\end{aligned}
$$

Here $\theta_{R}(t)$ is the time-dependent target leverage ratio, and the terms $-\lambda_{V}(t) \sigma_{V}(t)$ and $-\lambda_{Q}(t)$ $\sigma_{Q}(t)$ are included to account for the risk premiums of the firm's value and liability. Under the risk-neutral measure, $\delta$ is equal to zero. To obtain reasonable parameter values for the target leverage ratio, the dynamics of $Q$ and $V$ are under the actual measure such that $\delta$ is non-zero. If the leverage ratio $R$ and its associated model parameters in Eq. (3) could be directly observed in the market, Eq. (2) is still applicable to the model.

When the firm's leverage ratio is above a predefined level $R_{0}$, bankruptcy occurs before maturity. This is consistent with the event of bankruptcy being associated with abnormally high levels of debt relative to the market value of the firm's assets. As shown in the Appendix, the corresponding PD, $P_{\text {def }}(x, t)$, of a corporate discount bond over a period of time $t$ based on Eq. (2) can be approximated by

$$
\begin{aligned}
P_{\mathrm{def}}(x, t)=1 & -\int_{-\infty}^{0} \mathrm{~d} y\{G(x \exp [\alpha(t)], t ; y, 0)-G(x \exp [\alpha(t)], t ;-y, 0) \cdot \exp (\cdot 4 \beta y)\} \\
=1 & -N\left(-\frac{1}{\sqrt{2 c_{1}(t)}}\left[\exp (\alpha(t))+c_{2}(t)\right]\right) \\
& +N\left(\frac{1}{\sqrt{2 c_{1}(t)}}\left[x \exp (\alpha(t))+c_{2}(t)+8 \beta c_{1}(t)\right]\right) \\
& \times \exp \left\{4 \beta\left[x \exp (\alpha(t))+c_{2}(t)\right]+16 \beta^{2} c_{1}(t)\right\},
\end{aligned}
$$


where $x=\ln \left(R / R_{0}\right), N(\cdot)$ is the normalised cumulative distribution function,

$$
\begin{aligned}
& G(x \exp [\alpha(t)], t ; y, 0)=\frac{1}{\sqrt{4 \pi c_{1}(t)}} \exp \left\{-\frac{\left[x \exp [\alpha(t)]-y+c_{2}(t)\right]^{2}}{4 c_{1}(t)}\right\}, \\
& c_{1}(t)=\frac{1}{2} \int_{0}^{t} \mathrm{~d} \xi \sigma_{R}^{2}(\xi) \exp [2 \alpha(\xi)] \\
& c_{2}(t)=\int_{0}^{t} \mathrm{~d} \xi F(\xi) \exp [\alpha(\xi)]
\end{aligned}
$$

with

$$
\begin{aligned}
& \alpha(t)=-\int_{0}^{t} \mathrm{~d} \xi \kappa_{Q}(\xi) \\
& F(t)=\kappa_{Q}(t)\left[\ln \theta_{R}(t)-\ln R_{0}\right]-\frac{1}{2} \sigma_{R}^{2}(t)+\rho_{R r}(t) \sigma_{R}(t) \sigma_{r}(t) c_{4}(t) \exp \left[c_{3}(t)\right] \\
& c_{3}(t)=-\int_{0}^{t} \mathrm{~d} \xi \kappa_{r}(\xi) \\
& c_{4}(t)=-\int_{0}^{t} \mathrm{~d} \xi \exp \left[-c_{3}(\xi)\right] .
\end{aligned}
$$

The parameter $\beta$ is adjusted such that the approximate solution in Eq. (4) provides the best approximation to the exact result. A simple and easy-to-use method that has been developed by Lo, Lee, and Hui (2003) for solving barrier option values with time-dependent model parameters is provided for computing accurate PD estimates based on Eq. (4).

\section{Numerical results of model PD}

The computed PDs within a period of 15 years based on Eq. (4) for corporates with noninvestment ratings (i.e. CCC, B, and BB) are presented in Figs. 1, 2 and 3, respectively, and those with investment ratings (i.e. AAA, AA, A and BBB) are presented in Table 1. The model PDs of different periods of time are compared with the cumulative default rates of the corresponding ratings and periods of time based on 9769 companies' assigned long-term ratings from 1981 to 2001 reported by S\&P's (2002). The PDs generated from the CG model with a constant $\theta_{R}$ of 0.315 (i.e. the average leverage ratio of BBB-rated firms) and the Merton model are also shown in the figures and Table 1 for comparison. The predefined default-triggering level is set at $R_{0}=1$. The model parameters used for individual ratings are shown in Table 2. As $\sigma_{Q}$ of individual ratings is not well studied empirically in the literature, the use of $\sigma_{Q}=0.1$ for the calculations is based on the assumption that the volatility of a firm's liability structure would generally be lower than that of its firm value. If the leverage ratio $R$ and its associated model parameters in Eq. (4) could be directly observed in the market, it is not necessary to estimate $\sigma_{Q}$ empirically. Other common parameters used in the calculations are $\sigma_{r}=0.03162, \kappa_{r}=1.0$, $\rho_{V Q}=0.0, \rho_{V r}=0.0$ and $\rho_{Q r}=0.0$.

We use the simplest, yet non-trivial, scenario about the time-dependence of the target leverage ratio $\theta_{R}(t)$, namely a generic linear function of time with no adjustable parameter as follows:

$$
\theta_{R}(t)=\theta_{R 0}(1-\eta t)
$$

where $\theta_{R 0}$ and $\eta$ are constants such that:

1. in the first year $\theta_{R} \approx 0.732$ (i.e. the average leverage ratio of a corporate of 'CCC' rating), and

2. in the fifteenth year $\theta_{R} \approx 0.315$ (i.e. the average leverage ratio of a corporate of 'BBB' rating). 


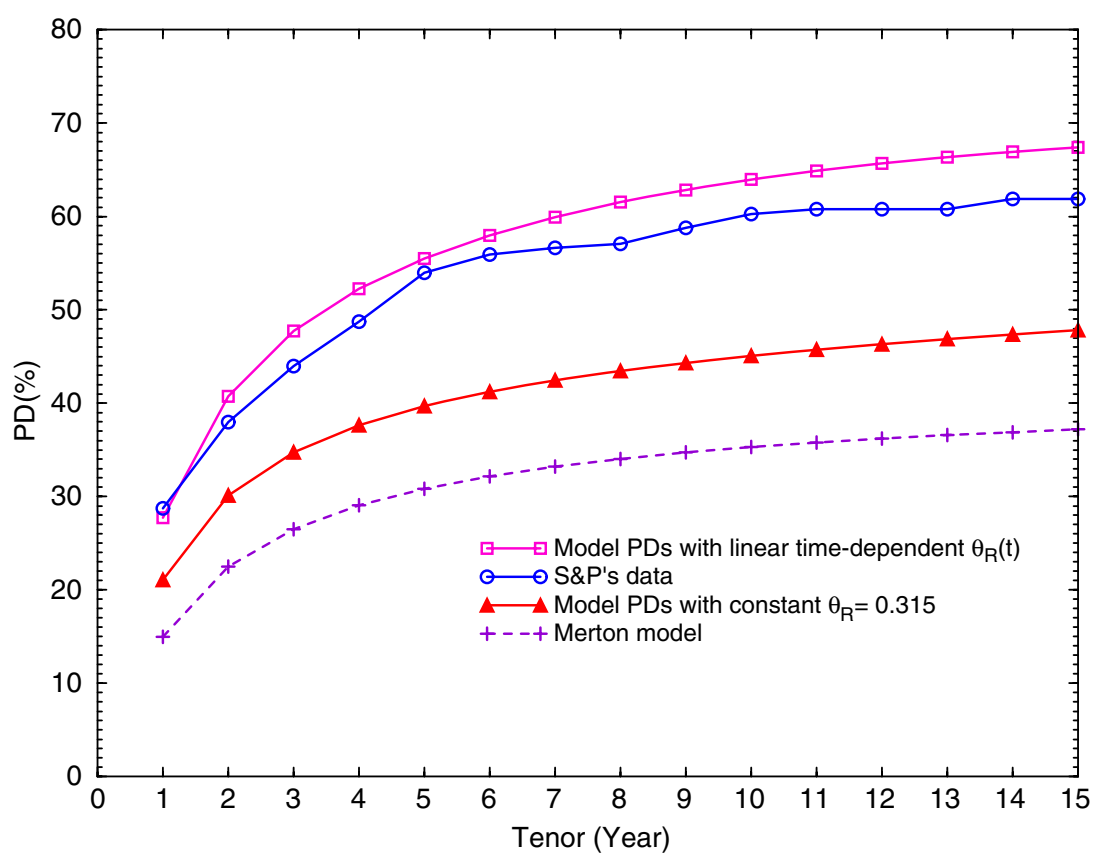

Fig. 1. PD term structures of CCC-rated firm with linear time-dependent target leverage ratio. The PDs of a CCC-rated firm with $R=73.2 \%$ and $\sigma_{V}=0.299$ are plotted using linear time-dependent $\theta_{R}(t)$. The other parameters used are $\kappa_{Q}=0.1, \sigma_{Q}=0.1, \sigma_{r}=0.0316, r=5 \%, \theta_{r}=5 \%, \kappa_{r}=1, \rho_{V Q}=0, \rho_{V r}=0$ and $\rho_{Q r}=0$. The term structures based on S\&P's (2002) data, the CG model with constant $\theta_{R}=0.315$ and Merton model with the corresponding rating and parameters are illustrated for comparison.

The simple linear function of time in Eq. (8) is used to illustrate the effect of the timedependence of a target leverage ratio on model PDs. The use of $\theta_{R}=0.315$ in the fifteenth year is close to the long-term target leverage ratio observed empirically by Collin-Dufresne and Goldstein (2001). The use of the high $\theta_{R}$ of 0.732 in the first year is explained by the observation that default occurring at short terms is mainly triggered by a corporate's short-term liabilities. The default (or distressed restructuring of debts) occurring in the corporate is due to its liquidity problem. The observation of the liquidity problem is similar to the phenomenon of high default rates of bonds at short maturities, that is called "crisis-at-maturity" by Johnson (1965). This explanation assumes, as Johnson points out, that corporates are unable to accumulate cash for debt repayment before maturity. The material one- to three-year default rates reported by S\&P's reflect the liquidity problem faced by the defaulted corporates in S\&P's data pool. The potential liquidity problem faced by corporates may cause their firm values to decline. The corporates may need to issue debts to repay their short-term obligations. The effect forces the corporates (including those corporates having low current leverage ratios) to observe high shortterm target leverage ratios.

The term structures of PDs for the CCC rating based on the three models, i.e. the timedependent model, CG model and Merton model, exhibit upward slopes at short tenors in Fig. 1. At longer tenors, their shapes are flat. Intuitively, this is because the probability that the firm's leverage ratio reaches the default barrier increases over time. The flattened slopes at longer tenors however reflect that PDs of CCC-rated corporates will not increase significantly over time 


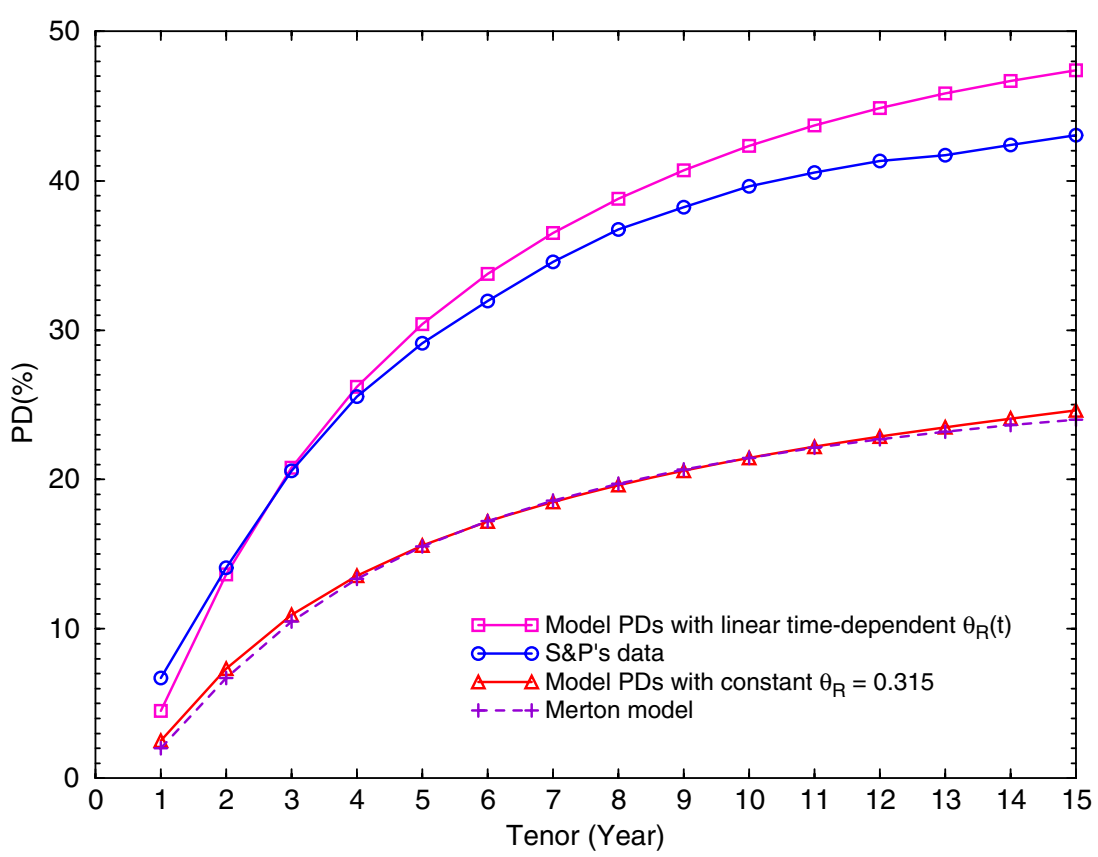

Fig. 2. PD term structures of B-rated firm with linear time-dependent target leverage ratio. The PDs of a B-rated firm with $R=53.8 \%$ and $\sigma_{V}=0.27$ are plotted using linear time-dependent $\theta_{R}(t)$. The other parameters used are $\kappa_{Q}=0.1, \sigma_{Q}=0.1$, $\sigma_{r}=0.0316, r=5 \%, \theta_{r}=5 \%, \kappa_{r}=1, \rho_{V Q}=0, \rho_{V r}=0$ and $\rho_{Q r}=0$. The term structures based on S\&P's (2002) data, the CG model with constant $\theta_{R}=0.315$ and Merton model with the corresponding rating and parameters are illustrated for comparison.

as the corporates survive at shorter terms. The shapes of the model term structures of PDs are consistent with S\&P's default rates of CCC-rated corporates. Fig. 1 shows that the PDs obtained from the time-dependent model and CG model are both higher than the PDs obtained from the Merton model which only considers default at maturity. In addition, the PDs based on the timedependent model are higher than those obtained from the CG model and broadly match with the default rates reported by S\&P's. The results demonstrate that the use of the high initial target leverage ratio of $\theta_{R}=0.732$, which gradually decreases to $\theta_{R}=0.315$ with time, can generate the model PDs which are more consistent with the empirical observed PDs than the PDs obtained from the CG and Merton models. In other words, the phenomenon of "crisis-at-maturity" that is presented by the high initial target leverage ratio could be a factor to explain the term structures of empirical default rates.

The term structures of PDs for the B rating are illustrated in Fig. 2 using the three models. The depicted term structures exhibit upward slopes at short tenors. At longer tenors, their shapes display different degrees of upward-sloping depending on the models. The shape and values of the term structure based on the time-dependent model match with the empirical default rates reported by S\&P's while the model values are higher than the empirical values at tenors of longer than four years. The values of the term structures generated from the other two models are close to each other but are much lower than the empirical values at all tenors. Similar to Fig. 1, Fig. 2 also shows that the use of the time-dependent target leverage ratio with linear time decay can generate the model PDs which are consistent with the empirical default rates.

Fig. 3 shows the term structures of PDs for the BB rating. The depicted term structures based on the time-dependent model exhibit upward slopes. The shape is consistent with the empirical 


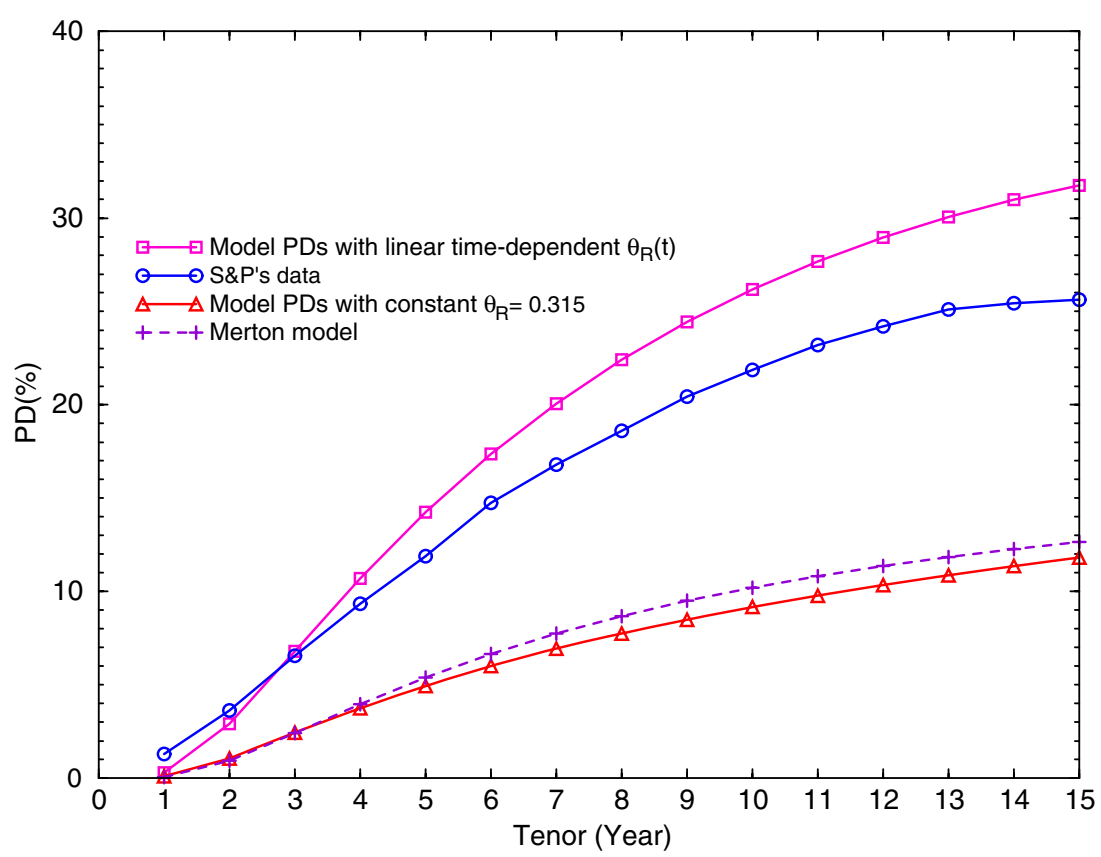

Fig. 3. PD term structures of BB-rated firm with linear time-dependent target leverage ratio. The PDs of a BB-rated firm with $R=49.5 \%$ and $\sigma_{V}=0.241$ are plotted using linear time-dependent $\theta_{R}(t)$. The other parameters used are $\kappa_{Q}=0.1$, $\sigma_{Q}=0.1, \sigma_{r}=0.0316, r=5 \%, \theta_{r}=5 \%, \kappa_{r}=1, \rho_{V Q}=0, \rho_{V r}=0$ and $\rho_{Q r}=0$. The term structures based on S\&P's (2002) data, the CG model with constant $\theta_{R}=0.315$ and Merton model with the corresponding rating and parameters are illustrated for comparison.

findings of the term structure of the BB rating reported by S\&P's, while the model term structure gives higher values of PDs in particular at tenors of longer than three years. The difference in the values increases with the tenors. The results indicate that the time-dependent target leverage ratio could be too high at longer tenors and gives higher model PDs than the empirical default rates. Conversely, the CG and Merton models present much lower PDs than the empirical default rates at all tenors, where the PDs given by the CG model are lower than those given by the Merton model. The constant target leverage ratio of $\theta_{R}=0.315$ used in the CG model could reduce the default risk as it is lower than the current leverage ratio of $R=0.495$ used for the $\mathrm{BB}$ rating.

As shown in the above numerical results, even though there are discrepancies between the model PDs and S\&P's data, yet the time-dependent model can qualitatively capture the major features of the historical default rates. The results support the hypothesis of the existence of timedependent target leverage ratios in firms with non-investment ratings. Furthermore, to examine the possibility of different time-dependent target leverage ratios for different ratings, we use a family of time-dependent ratios parameterised by a single parameter $\gamma$ and try to determine the values of $\gamma$ for different ratings by fitting the historical default rates with the model PDs. The time-dependent target leverage ratio $\theta_{R}(t)$ is assumed to be parameterised in the following exponential form:

$$
\theta_{R}(t)=\theta_{R 0}[1+\eta \exp (-\gamma t)]
$$

where $\theta_{R 0}, \eta$ and $\gamma$ are constants determined by fitting the numerical estimates with the average default rates reported by S\&P's. The conditions of $\theta_{R}(t=1) \approx 0.732$ and $\theta_{R}(t=15) \approx 0.315$ 
Table 1

PDs (in \%) of investment-rated firms with exponential and linear time-dependent target leverage ratios

\begin{tabular}{|c|c|c|c|c|c|c|c|c|c|c|c|c|c|c|c|c|}
\hline Rating & Models & Yr. 1 & Yr. 2 & Yr. 3 & Yr. 4 & Yr. 5 & Yr. 6 & Yr. 7 & Yr. 8 & Yr. 9 & Yr. 10 & Yr. 11 & Yr. 12 & Yr. 13 & Yr. 14 & Yr. 15 \\
\hline \multirow[t]{5}{*}{ AAA } & S\&P's & .00 & 0.00 & .03 & 0.07 & .11 & .21 & 0.31 & 0.49 & 55 & 0.63 & 0.63 & 0.63 & 0.63 & 0.63 & 0.63 \\
\hline & Model (exponential) & 0.0000 & 0.0000 & 0.0000 & 0.0000 & 0.0016 & 0.0214 & 0.0969 & 0.2387 & 0.4063 & 0.5476 & 0.6374 & 0.6806 & 0.6959 & 0.6996 & 0.7001 \\
\hline & Model (linear) & 0.0000 & 0.0000 & 0.0000 & 0.0000 & 0.0004 & 0.0049 & 0.0194 & 0.0420 & 0.0638 & 0.0786 & 0.0861 & 0.0891 & 0.0900 & 0.0902 & 0.0903 \\
\hline & CG & 0.0000 & 0.0000 & 0.0000 & 0.0000 & 0.0000 & 0.0000 & 0.0000 & 0.0000 & 0.0000 & 0.0000 & 0.0000 & 0.0000 & 0.0000 & 0.0000 & 0.0000 \\
\hline & Merton & 0.0000 & 0.0000 & 0.0000 & 0.0000 & 0.0000 & 0.0000 & 0.0000 & 0.0000 & 0.0000 & 0.0000 & 0.0000 & 0.0000 & 0.0000 & 0.0000 & 0.0000 \\
\hline \multirow[t]{5}{*}{ AA } & S\&P's & 0.01 & 0.03 & 0.09 & 0.16 & 0.26 & 0.39 & 0.56 & 0.71 & 0.82 & 0.99 & 1.14 & 1.32 & 1.43 & 1.56 & 1.72 \\
\hline & Model ( & 0.0000 & 0.0000 & 0.0000 & 0.0020 & 0.0255 & 0.1201 & 0.3172 & 0.5890 & 0.8735 & 1.1174 & 1.2961 & 1.4098 & 1.4729 & 1.5030 & 1.5152 \\
\hline & Model (linear) & 0.0000 & 0.0000 & 0.0000 & 0.0017 & 0.0221 & 0.1021 & 0.2656 & 0.4864 & 0.7130 & 0.9037 & 1.0410 & 1.1274 & 1.1750 & 1.1980 & 1.2076 \\
\hline & $\mathrm{CG}$ & 0.0000 & 0.0000 & 0.0000 & 0.0000 & 0.0000 & 0.0000 & 0.0000 & 0.0000 & 0.0000 & 0.0001 & 0.0002 & 0.0002 & 0.0003 & 0.0004 & 0.0006 \\
\hline & Merton & 0.0000 & 0.0000 & 0.0000 & 0.0000 & 0.0000 & 0.0000 & 0.0000 & 0.0000 & 0.0000 & 0.0000 & 0.0000 & 0.0001 & 0.0001 & 0.0002 & 0.0003 \\
\hline \multirow[t]{5}{*}{ A } & S\&P's & 0.05 & 0.14 & 0.26 & 0.43 & 0.64 & 0.85 & 1.08 & 1.33 & 1.62 & 1.90 & 2.11 & 2.30 & 2.49 & 2.66 & 2.98 \\
\hline & Model (exponential) & 0.0000 & 0.0001 & 0.0075 & 0.0629 & 0.2208 & 0.4939 & 0.8483 & 1.2327 & 1.6034 & 1.9326 & 2.2081 & 2.4286 & 2.5990 & 2.7271 & 2.8214 \\
\hline & Model (linear) & 0.0000 & 0.0001 & 0.0099 & 0.0891 & 0.3347 & 0.7949 & 1.4368 & 2.1796 & 2.9360 & 3.6369 & 4.2395 & 4.7250 & & 5.3555 & 5.5308 \\
\hline & CG & 0.0000 & 0.0000 & 0.0000 & 0.0004 & 0.0015 & 0.0040 & 0.0082 & 0.0142 & 0.0220 & 0.0315 & 0.0425 & 0.0549 & 0.0683 & 0.0828 & 0.0981 \\
\hline & Merton & 0.0000 & 0.0000 & 0.0000 & 0.0003 & 0.0015 & 0.0048 & 0.0110 & 0.0209 & 0.0344 & 0.0515 & 0.0716 & 0.0943 & 0.1191 & 0.1454 & 0.1728 \\
\hline \multirow[t]{5}{*}{ BBB } & S\&P's & 0.27 & 0.62 & 0.99 & 1.63 & 2.26 & 3.00 & 3.67 & 4.27 & 4.76 & 5.35 & 6.03 & 6.53 & 7.06 & 7.71 & 8.37 \\
\hline & Model (expon & 0.0012 & 0.1051 & 0.5298 & 1.2263 & 2.0441 & 2.8753 & 3.6650 & 4.3918 & 5.0519 & 5.6492 & 6.1904 & 6.6831 & 7.1343 & 7.5502 & 7.9360 \\
\hline & Model (linear) & 0.0013 & 0.1306 & 0.7232 & 1.8194 & 3.2563 & 4.8621 & 6.5093 & 8.1140 & 9.6246 & 11.0115 & 12.2596 & 13.3631 & 14.3225 & 15.1425 & 15.8304 \\
\hline & $\mathrm{CG}$ & 0.0003 & 0.0274 & 0.1449 & 0.3552 & 0.6283 & 0.9367 & 1.2617 & 1.5919 & 1.9206 & 2.2443 & 2.5609 & 2.8697 & 3.1706 & 3.4637 & 3.7494 \\
\hline & Merton & 0.0001 & 0.0146 & 0.1032 & 0.2906 & 0.5555 & 0.8670 & 1.1999 & 1.5370 & 1.8670 & 2.1835 & 2.4828 & 2.7630 & 3.0237 & 3.2651 & 3.4877 \\
\hline
\end{tabular}

The PDs of investment rated firms are computed using exponential and linear time-dependent $\theta_{R}(t)$, respectively, where $\gamma(\mathrm{BBB})=-0.31, \gamma(\mathrm{A})=-0.09, \gamma(\mathrm{AA})=0.0165$ and $\gamma(\mathrm{AAA})=0.097$. The parameters used are those in Table $2, \sigma_{Q}=0.1, \sigma_{r}=0.0316, r=5 \%, \theta_{r}=5 \%, \kappa_{r}=1, \rho_{V Q}=0, \rho_{V r}=0$ and $\rho_{Q r}=0$. The PDs based on S\&P's $(2002)$ data, the CG model with constant $\theta_{R}=0.315$ and the Merton model with the corresponding ratings and parameters are illustrated for comparison. 
Table 2

Parameters used in the calculations for individuals ratings

\begin{tabular}{llllllll}
\hline & AAA & AA & A & BBB & BB & B & CCC \\
\hline Leverage ratio $R(\%)$ & 3.1 & 9.5 & 17.2 & 31.5 & 49.5 & 53.8 & 73.2 \\
Volatility of the firm value $\sigma_{V}$ & 0.127 & 0.156 & 0.184 & 0.213 & 0.241 & 0.270 & 0.299 \\
Speed of mean-reverting $\kappa_{Q}$ & 0.4 & 0.3 & 0.2 & 0.1 & 0.1 & 0.1 & 0.1 \\
\hline
\end{tabular}

The leverage ratios $R$ are based on the industry medians reported by S\&P's (2001). The values of $\sigma_{V}$ fall close to the estimates of Delianedis and Geske (1999). The values of $\kappa_{Q}$ are based on the estimates of Fama and French (1999), $\left(\kappa_{Q} \approx 0.1\right)$, who investigate the universe of firms and Shyam-Sunder and Myers $(1999),\left(\kappa_{Q} \approx 0.4\right)$, whose sample includes large and financially conservative firms (note: $\sigma_{R}=\sqrt{\sigma_{V}^{2}+\sigma_{Q}^{2}-2 \rho_{V Q} \sigma_{Q} \sigma_{V}}$ ).

will enable us to express $\theta_{R 0}$ and $\eta$ as functions of $\gamma$. In other words, we are left with the parameter $\gamma$ only. In our search for optimal values of $\gamma$ such that model PDs fit the empirical values, we find that $\gamma=-0.23,-0.1$ and -0.15 for ratings of $\mathrm{CCC}, \mathrm{B}$ and $\mathrm{BB}$, respectively. Using these values of $\gamma$, the decrease in the target leverage ratio with time is faster than that based on the linear function. In Fig. 4, the model PDs generated from the exponential timedependent function of the target leverage ratio match more closely with the empirical values at all tenors than the model PDs presented in Figs. 1,2 and 3. The results also provide evidence to support the hypothesis of the existence of a time-dependent target leverage ratio. As the values of $\gamma$ are found to be different for different ratings, firms with different credit quality may have different paces to move their initial target ratios toward the long-term target ratio over time.

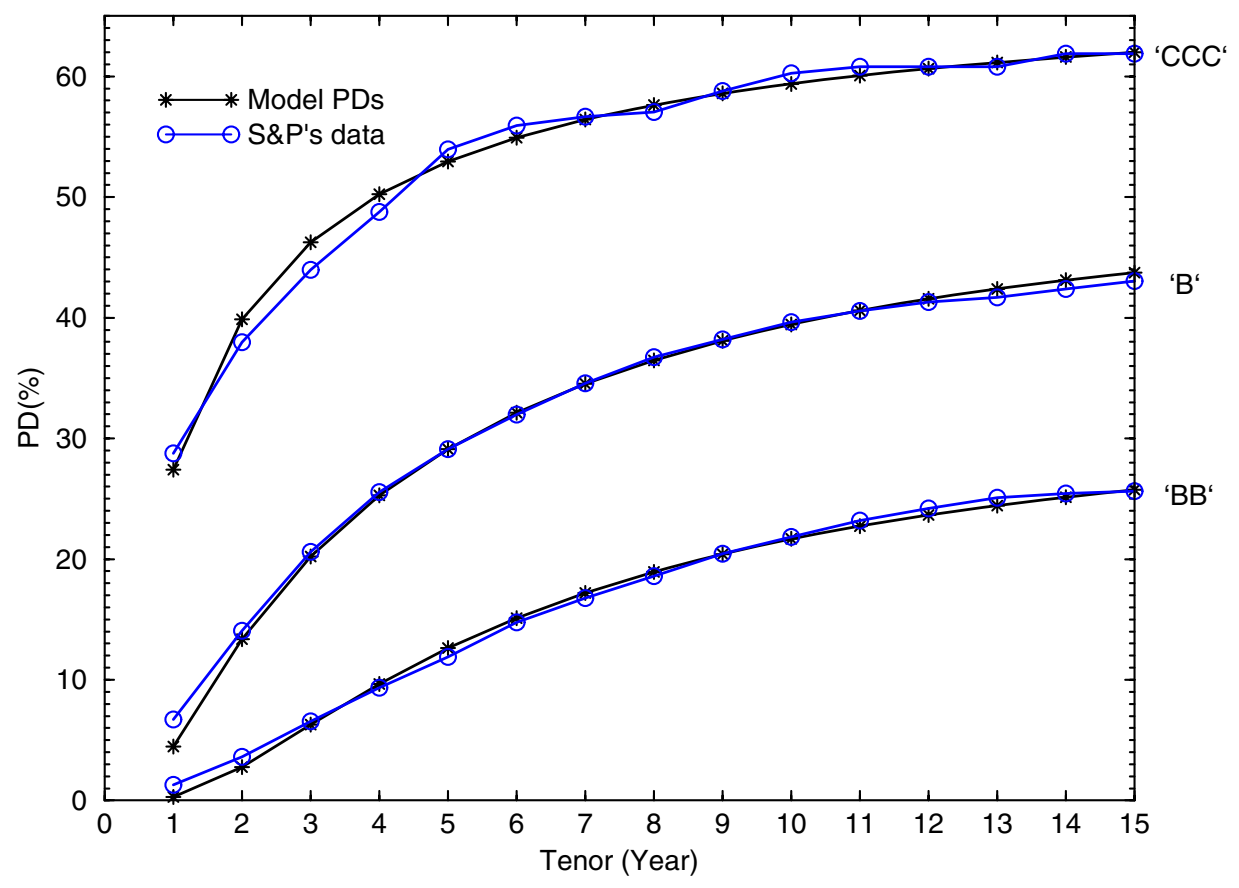

Fig. 4. PD term structures of non-investment rated firms with exponential time-dependent target leverage ratio. The PDs of non-investment $(\mathrm{CCC}, \mathrm{B}$ and $\mathrm{BB})$ rated firms are plotted using exponential time-dependent $\theta_{R}(t)$, where $\gamma(\mathrm{CCC})=-0.23, \gamma(\mathrm{B})=-0.1$ and $\gamma(\mathrm{BB})=-0.15$. The other parameters used are $\kappa_{Q}=0.1, \sigma_{Q}=0.1, \sigma_{r}=0.0316, r=5 \%$, $\theta_{r}=5 \%, \kappa_{r}=1, \rho_{V Q}=0, \rho_{V r}=0$ and $\rho_{Q r}=0$. The term structures based on S\&P's (2002) data with the corresponding ratings are illustrated for comparison. 
However, it is beyond the scope of this paper to investigate the actual time-dependence of different firms' target leverage ratios.

In Table 1, the PDs generated by the time-dependent model based on the exponential function of $\theta_{R}(t)$ for investment ratings are comparable to S\&P's default rates, while those based on the linear function do not match well with the empirical values. The values of the term structure of the $\mathrm{BBB}$ rating generated by the time-dependent model with the exponential function of $\theta_{R}(t)$ broadly match with the empirical default rates reported by S\&P's. The percentage differences between the model PDs and empirical default rates for investment ratings (in particular for AAA and AA ratings at short tenors) are larger than those for non-investment ratings. The first reason is that the observations of default events occurred in investment grade firms are rare, in particular at short tenors (e.g. only 14 defaults occurred in companies with original rating of AA in S\&P's data and the average time to default is 11.9 years). A very few default events which occurred in AA-rated firms may cause significant changes of the observed default rates. On the other hand, there are 574 defaults which occurred in firms with the original rating of B in S\&P's data and the average time to default is only 3.8 years. The second reason is that the problem of downwardbiased default risk at short tenors is common to all contingent-claims pricing models which assume continuous dynamics. Therefore, criticism of the model based on low PDs at short tenors is that of the underlying assumptions. Regarding PDs generated by the CG and Merton models, the prediction by these two models that PDs are close to zero at all tenors for the AAA, AA and A ratings contradicts the findings of S\&P's (2002).

We use the BB rating to illustrate the effect of the correlation $\rho_{V Q}, \rho_{V r}$ and $\rho_{Q r}$ on model PDs in Figs. 5, 6 and 7, respectively. The movement of the target leverage ratio follows the linear

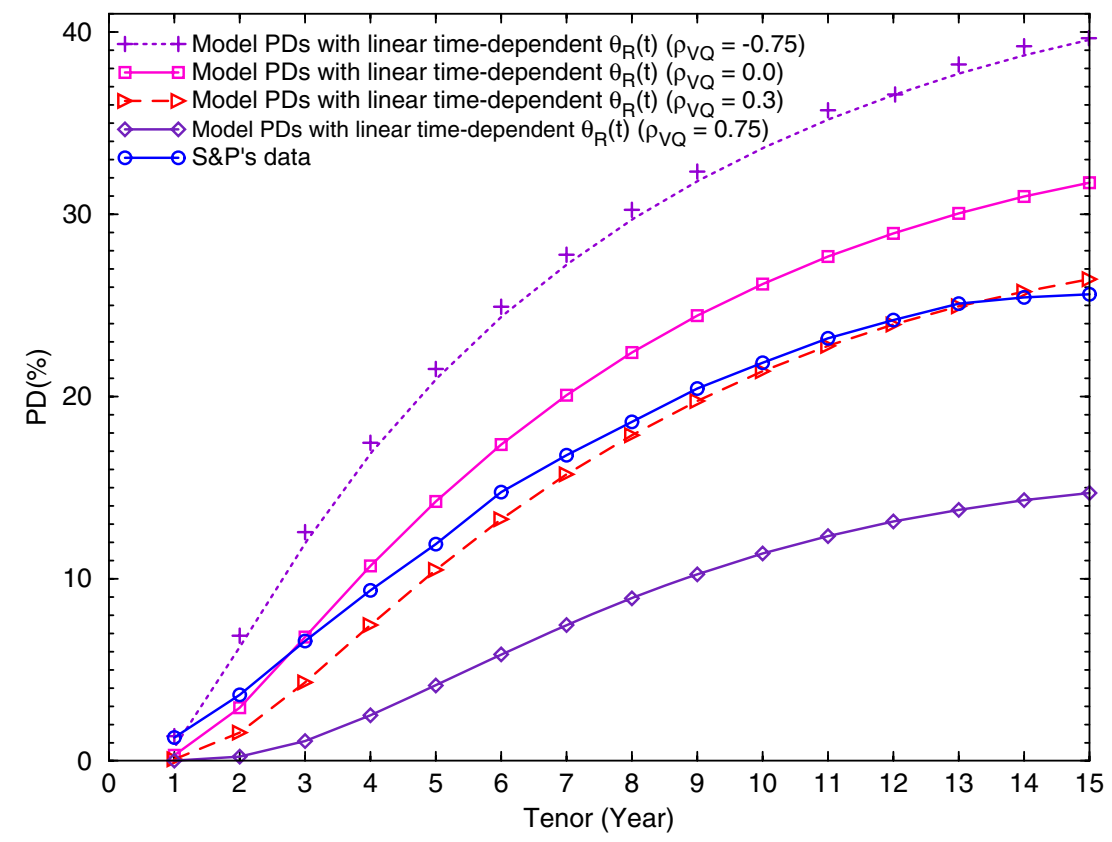

Fig. 5. PD term structures of BB-rated firm with different $\rho_{V Q}$ and linear time-dependent target leverage ratio. The PDs of a BB-rated firm with $R=49.5 \%$ and $\sigma_{V}=0.241$ are plotted using $\rho_{V Q}=-0.75,0,0.3$ and 0.75 and linear time-dependent $\theta_{R}(t)$. The other parameters used are $\kappa_{Q}=0.1, \sigma_{Q}=0.1, \sigma_{r}=0.0316, r=5 \%, \theta_{r}=5 \%, \kappa r=1, \rho_{V r}=0$ and $\rho_{Q r}=0$. The term structure based on S\&P's (2002) data with the corresponding rating is illustrated for comparison. 


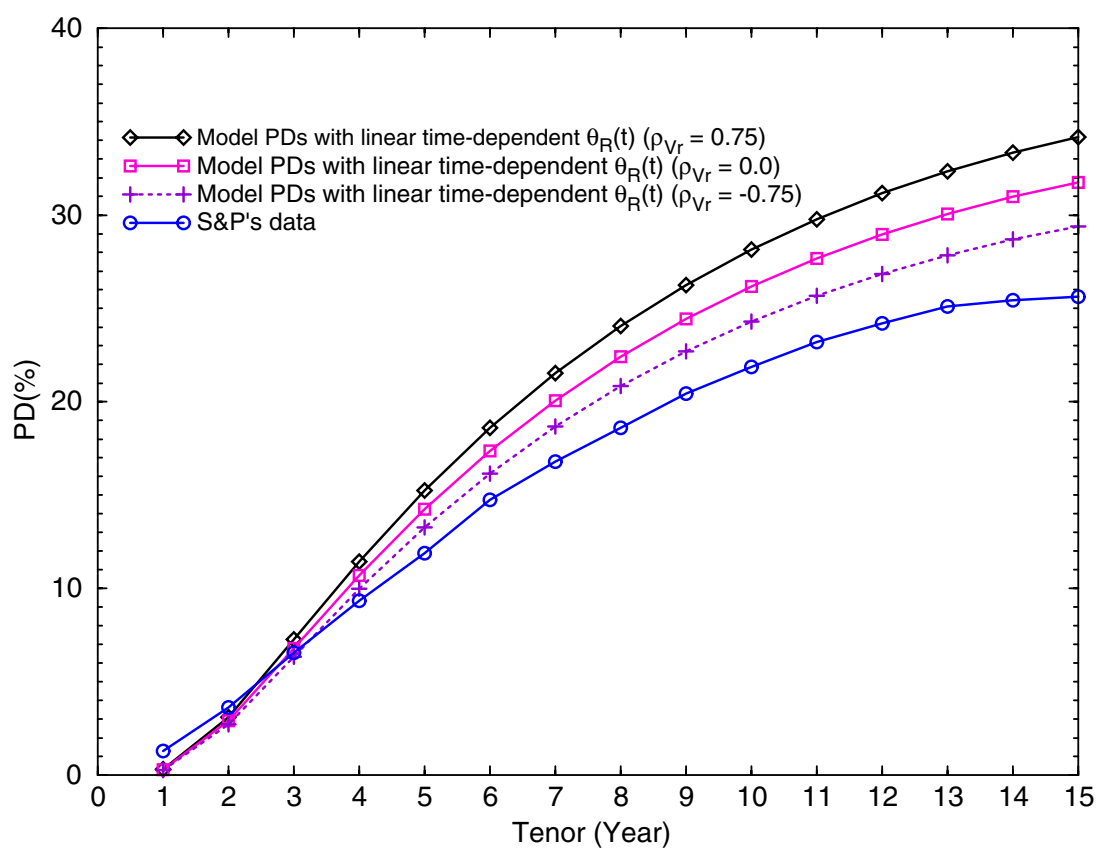

Fig. 6. PD term structures of BB-rated firm with different $\rho_{V r}$ and linear time-dependent target leverage ratio. The PDs of a BB-rated firm with $R=49.5 \%$ and $\sigma_{V}=0.241$ are plotted using $\rho_{V r}=-0.75,0$ and 0.75 and linear time-dependent $\theta_{R}$ $(t)$. The other parameters used are $\kappa_{Q}=0.1, \sigma_{Q}=0.1, \sigma_{r}=0.0316, r=5 \%, \theta_{r}=5 \%, \kappa_{r}=1, \rho_{V Q}=0$ and $\rho_{Q r}=0$. The term structure based on S\&P's (2002) data with the corresponding rating is illustrated for comparison.

function in Eq. (8). In Fig. 5, $\rho_{V Q}=-0.75,0,0.3$ and 0.75 are used to generate the term structures of PDs. The values of PDs increase with the decrease in $\rho_{V Q}$ and are sensitive to $\rho_{V Q}$. This is due to the reason that the opposite movement of $V$ and $Q$ caused by negative correlation would further increase the leverage ratio of the firm (when $V$ decreases or $Q$ increases) so as to increase the default risk. Conversely, when $Q$ and $V$ are positively correlated, the expected leverage ratio would be more stable over time and thus the default risk is reduced. Fig. 5 also illustrates that different $\rho_{V Q}$ can produce quite diverse shapes of the term structures. The use of $\rho_{V Q}=0.3$ generates the term structure which matches the S\&P's data better than that generated by the use of $\rho_{V Q}=0$. This implies that firms may generally intend to maintain positive correlation between its firm asset and liability. This can be explained by the intuition that a firm tends to borrow more money for expanding its business when its firm value increases. When its firm value drops, it tends to reduce its debt and also its expenses. As a result, correlation between the firm's asset and liability values is positive.

Figs. 6 and 7 show that the impact of the correlation arising from $r$, in particular the impact from $\rho_{Q r}$, on PDs is not as significant as that from $\rho_{V Q}$. The impact only becomes apparent at longer tenors. It is observed in Fig. 6 that PDs decrease with the decrease in $\rho_{V r}$. The reason is that PDs would increase due to the decrease in the drift $r$ of the asset value $V$. As negative $\rho_{V r}$ implies that a decrease in $r$ would relate to an increase in $V$, this provides a natural hedge against default risk. This observation is also consistent with the intuition that a decrease in $r$ would typically be associated with an increase in $V$ and PDs may thus decrease. Conversely, Fig. 7 shows that PDs increase with a decrease in $\rho_{Q r}$. As PDs would increase due to the increase in the drift $r$ of the liability $Q$, negative $\rho_{Q r}$ reinforces this effect and increases the default risk. This 


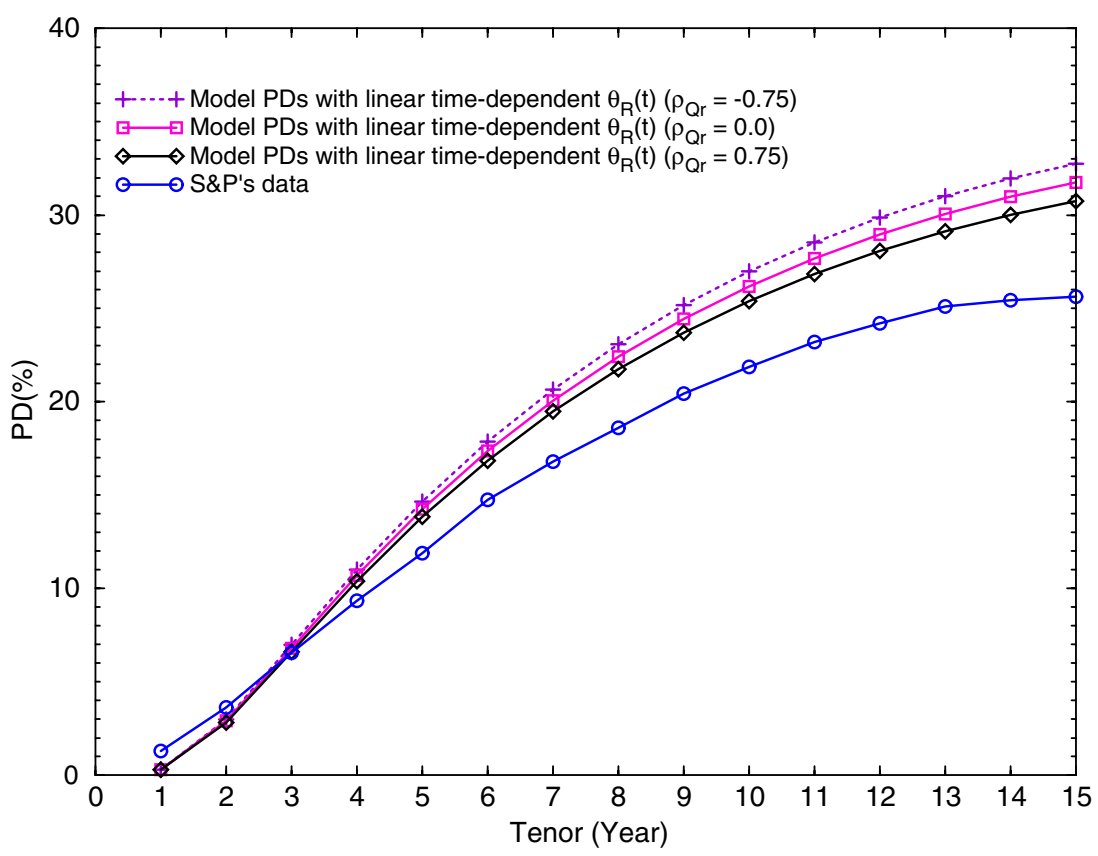

Fig. 7. PD term structures of BB-rated firm with different $\rho_{Q r}$ and linear time-dependent target leverage ratio. The PDs of a BB-rated firm with $R=49.5 \%$ and $\sigma_{V}=0.241$ are plotted using $\rho_{Q r}=-0.75,0$ and 0.75 and linear time-dependent $\theta_{R}$ $(t)$. The other parameters used are $\kappa_{Q}=0.1, \sigma_{Q}=0.1, \sigma_{r}=0.0316, r=5 \%, \theta_{r}=5 \%, \kappa_{r}=1, \rho_{V Q}=0$ and $\rho_{V r}=0$. The term structure based on S\&P's (2002) data with the corresponding rating is illustrated for comparison.

observation is consistent with the intuition that a low interest rate environment would encourage firms to increase their debts because of low interest rate costs involved and the firms' PDs would thus increase.

In summary, the numerical results show that the stationary-leverage-ratio model incorporating a time-dependent target leverage ratio gives the basic shapes and values of the term structures of PDs for different credit ratings, in particular for ratings of BBB and below, which broadly match with some empirical findings. On the other hand, the PDs obtained from the CG and Merton models are much lower than the empirical values. The numerical results provide some evidences of the existence of a time-dependent target leverage ratio.

\section{Conclusion}

This paper extends the stationary-leverage-ratio model to incorporate a time-dependent target leverage ratio. The numerical results show that the model incorporating a timedependent target leverage ratio is capable of producing term structures of PDs which are consistent with some empirical findings. The results provide evidence to support the hypothesis of the existence of a time-dependent target leverage ratio. The hypothesis reflects the movement of a firm's initial target ratio toward a long-term target ratio over time. As the scenarios about the time-dependence of target leverage ratios used in this paper are simply for illustrative purposes, more detailed empirical examination on the actual time-dependence of target leverage ratios and comparison between the empirical default rates and model PDs are left to future research. 


\section{Acknowledgement}

This work is partially supported by the Direct Grant for Research from the Research Grants Council of the Hong Kong Government. The conclusions herein do not represent the views of the Hong Kong Monetary Authority.

\section{Appendix A}

Applying the Ito's lemma and the standard risk-neutral argument, the price $P$ of a corporate bond with stochastic interest rate and liability, which is a function of the firm value $V$, the liability $Q$ of the firm, the short-term interest rate $r$ and the time-to-maturity $t$ is governed by the partial differential equation

$$
\begin{aligned}
\frac{\partial P(Q, V, r, t)}{\partial t}= & \frac{1}{2} \sigma_{Q}^{2}(t) Q^{2} \frac{\partial^{2} P}{\partial Q^{2}}+\frac{1}{2} \sigma_{V}^{2}(t) V^{2} \frac{\partial^{2} P}{\partial V^{2}}+\frac{1}{2} \sigma_{r}^{2}(t) \frac{\partial^{2} P}{\partial r^{2}} \\
& +\rho_{Q V}(t) \sigma_{Q}(t) \sigma_{V}(t) Q V \frac{\partial^{2} P}{\partial Q \partial V}+\rho_{Q r}(t) \sigma_{Q}(t) \sigma_{r}(t) Q \frac{\partial^{2} P}{\partial Q \partial r} \\
& +\rho_{V r}(t) \sigma_{V}(t) \sigma_{r}(t) V \frac{\partial^{2} P}{\partial V \partial r}+\left\{r-\lambda_{V}(t) \sigma_{V}(t)\right\} V \frac{\partial P}{\partial V} \\
& +\left\{r-\lambda_{Q}(t) \sigma_{Q}(t)+\kappa_{Q}(t)[\ln V-\ln Q]\right\} Q \frac{\partial P}{\partial Q} \\
& +\kappa_{r}(t)\left[\theta_{r}(t)-r\right] \frac{\partial P}{\partial r}-r P
\end{aligned}
$$

where the terms $-\lambda_{V}(t) \sigma_{V}(t)$ and $-\lambda_{Q}(t) \sigma_{Q}(t)$ are included to account for the risk premiums of the firm's value and liability. To solve this partial differential equation, we first rewrite it in terms of the variables $x_{1}=\ln \left(Q / Q_{0}\right)-\ln \left(V / V_{0}\right)$ and $x_{2}=\ln \left(Q / Q_{0}\right)+\ln \left(V / V_{0}\right)$, where $Q_{0}$ and $V_{0}$ are constants, as follows:

$$
\begin{aligned}
\frac{\partial P\left(x_{1}, x_{2}, r, t\right)}{\partial t}= & \frac{1}{2} \sigma_{1}^{2}(t) \frac{\partial^{2} P}{\partial x_{1}^{2}}+\frac{1}{2} \sigma_{2}^{2}(t) \frac{\partial^{2} P}{\partial x_{2}^{2}}+\frac{1}{2} \sigma_{r}^{2}(t) \frac{\partial^{2} P}{\partial r^{2}} \\
& +\rho_{12}(t) \sigma_{1}(t) \sigma_{2}(t) \frac{\partial^{2} P}{\partial x_{1} \partial x_{2}}+\rho_{1 r}(t) \sigma_{1}(t) \sigma_{r}(t) \frac{\partial^{2} P}{\partial x_{1} \partial r} \\
& +\rho_{2 r}(t) \sigma_{2}(t) \sigma_{r}(t) \frac{\partial^{2} P}{\partial x_{2} \partial r}+\left[\eta_{1}(t)-\frac{1}{2} \sigma_{1}^{2}(t)-\kappa_{Q}(t) x_{1}\right] \frac{\partial P}{\partial x_{1}} \\
& +\left[2 r+\eta_{2}(t)-\frac{1}{2} \sigma_{2}^{2}(t)-\kappa_{Q}(t) x_{1}\right] \frac{\partial P}{\partial x_{2}}+\kappa_{r}(t)\left[\theta_{r}(t)-r\right] \frac{\partial P}{\partial r} \\
& -r P
\end{aligned}
$$


where

$$
\begin{aligned}
& \sigma_{1}=\sqrt{\sigma_{Q}^{2}+\sigma_{V}^{2}-2 \rho_{Q V} \sigma_{Q} \sigma_{V}}, \quad \sigma_{2}=\sqrt{\sigma_{Q}^{2}+\sigma_{V}^{2}+2 \rho_{Q V} \sigma_{Q} \sigma_{V}} \\
& \rho_{12}=\frac{\sigma_{Q}^{2}-\sigma_{V}^{2}}{\sigma_{1} \sigma_{2}}, \quad \rho_{1 r}=\frac{\rho_{Q r} \sigma_{Q}-\rho_{V r} \sigma_{V}}{\sigma_{1}}, \quad \rho_{2 r}=\frac{\rho_{Q r} \sigma_{Q}+\rho_{V r} \sigma_{V}}{\sigma_{2}} \\
& \eta_{1}=\sigma_{V}^{2}-\rho_{Q V} \sigma_{Q} \sigma_{V}-\left(\lambda_{Q} \sigma_{Q}-\lambda_{V} \sigma_{V}\right)-\kappa_{Q} \ln \left(\frac{Q_{0}}{V_{0}}\right), \\
& \eta_{2}=\rho_{Q V} \sigma_{Q} \sigma_{V}-\left(\lambda_{Q} \sigma_{Q}+\lambda_{V} \sigma_{V}\right)-\kappa_{Q} \ln \left(\frac{Q_{0}}{V_{0}}\right) .
\end{aligned}
$$

Since $P\left(x_{1}, x_{2}, r, 0\right)$ is independent of $r$ and $x_{2}$, it is not difficult to show that $P\left(x_{1}, x_{2}, r, t\right)$ actually does not depend upon $x_{2}$ and thus Eq. (A.2) is reduced to

$$
\begin{aligned}
\frac{\partial P\left(x_{1}, r, t\right)}{\partial t}= & \frac{1}{2} \sigma_{1}^{2}(t) \frac{\partial^{2} P}{\partial x_{1}^{2}}+\frac{1}{2} \sigma_{r}^{2}(t) \frac{\partial^{2} P}{\partial r^{2}}+\rho_{1 r}(t) \sigma_{1}(t) \sigma_{r}(t) \frac{\partial^{2} P}{\partial x_{1} \partial r} \\
& +\left[\eta_{1}(t)-\frac{1}{2} \sigma_{1}^{2}(t)-\kappa_{Q}(t) x_{1}\right] \frac{\partial p}{\partial x_{1}}+\kappa_{r}(t)\left[\theta_{r}(t)-r\right] \frac{\partial P}{\partial r}-r P,
\end{aligned}
$$

whose solution has the form

$$
P\left(x_{1}, r, t\right)=B(r, t) \mathcal{P}\left(x_{1}, t\right)
$$

where $\mathcal{P}\left(x_{1}, t\right)$ satisfies the partial differential equation

$$
\frac{\partial \mathcal{P}\left(x_{1}, t\right)}{\partial t}=\frac{1}{2} \sigma_{1}^{2}(t) \frac{\partial^{2} \mathcal{P}}{\partial x_{1}^{2}}+\left[\gamma(t)-\kappa_{Q}(t) x_{1}\right] \frac{\partial \mathcal{P}}{\partial x_{1}},
$$

and $B(r, t)=\exp \left\{A_{1}(t)+A_{2}(t) A_{3}^{2}(t) \exp \left[2 A_{4}(t)\right]+A_{3}(t) \exp \left[A_{4}(t)\right] r\right\}$ is simply the risk-free bond function of the Vasicek model with explicitly time-dependent parameters. Here the parameters $\gamma(t)$ and $A_{n}$ 's are defined as follows:

$$
\begin{aligned}
\gamma(t)= & \sigma_{V}^{2}-\rho_{Q V}(t) \sigma_{Q}(t) \sigma_{V}(t)-\lambda_{Q}(t) \sigma_{Q}(t)+\lambda_{V}(t) \sigma_{V}(t)-\kappa_{Q}(t) \ln \left(\frac{Q_{0}}{V_{0}}\right) \\
& +\rho_{1 r}(t) \sigma_{1}(t) \sigma_{r}(t) A_{3}(t) \exp \left[A_{4}(t)\right]-\frac{1}{2} \sigma_{1}^{2}(t), \\
A_{4}(t)= & -\int_{0}^{t} \kappa_{r}\left(t^{\prime}\right) \mathrm{d} t^{\prime} \\
A_{3}(t)= & -\int_{0}^{t} \exp \left[-A_{4}\left(t^{\prime}\right)\right] \mathrm{d} t^{\prime}, \\
A_{2}(t)= & \frac{1}{2} \exp \left[-2 A_{4}(t)\right] \int_{0}^{t} \sigma_{r}^{2}\left(t^{\prime}\right) \exp \left[2 A_{4}\left(t^{\prime}\right)\right] \mathrm{d} t^{\prime}, \\
A_{1}(t)= & \int_{0}^{t}\left[\kappa_{r}\left(t^{\prime}\right) \theta_{r}\left(t^{\prime}\right)+2 A_{2}\left(t^{\prime}\right)\right] A_{3}\left(t^{\prime}\right) \exp \left[A_{4}\left(t^{\prime}\right)\right] \mathrm{d} t^{\prime} .
\end{aligned}
$$


It should be noted that defining

$$
\begin{aligned}
& R \equiv Q / V=\left(Q_{0} / V_{0}\right) \exp \left(x_{1}\right), \\
& \ln \theta_{R}(t) \equiv \frac{1}{\kappa_{Q}}\left(\sigma_{V}^{2}-\rho_{Q V} \sigma_{Q} \sigma_{V}-\lambda_{Q} \sigma_{Q}+\lambda_{V} \sigma_{V}\right), \\
& \sigma_{R}(t)=\sigma_{1}(t), \quad \rho_{R r}(t)=\rho_{1 r}(t),
\end{aligned}
$$

we can straightforwardly derive Eq. (2) from Eq. (A.4). Obviously, Eq. (A.6) can be easily solved to yield

$$
\mathcal{P}\left(x_{1}, t\right)=\exp \left\{\alpha(t) x_{1} \frac{\partial}{\partial x_{1}}\right\} \tilde{P}\left(x_{1}, t\right)=\tilde{P}\left(x_{1} \exp \{\alpha(t)\}, t\right),
$$

where

$$
\alpha(t)=-\int_{0}^{t} \mathrm{~d} t^{\prime} \kappa_{Q}\left(t^{\prime}\right)
$$

and

$$
\tilde{P}\left(x_{1}, t\right)=\int_{-\infty}^{\infty} \mathrm{d} x_{1}^{\prime} G\left(x_{1}, t ; x_{1}^{\prime}, 0\right) \tilde{P}\left(x_{1}, 0\right) .
$$

The kernel $G\left(x_{1}, t ; x_{1}^{\prime}, 0\right)$ is given by

$$
G\left(x_{1}, t ; x_{1}^{\prime}, 0\right)=\frac{1}{\sqrt{4 \pi c_{1}(t)}} \exp \left\{-\frac{\left[x_{1}^{\prime}-x_{1}-c_{2}(t)\right]^{2}}{4 c_{1}(t)}\right\}
$$

where

$$
\begin{aligned}
& c_{1}(t)=\frac{1}{2} \int_{0}^{t} \sigma_{1}^{2}\left(t^{\prime}\right) \exp \left[2 \alpha\left(t^{\prime}\right)\right] \mathrm{d} t^{\prime}, \\
& c_{2}(t)=\int_{0}^{t} \gamma\left(t^{\prime}\right) \exp \left[\alpha\left(t^{\prime}\right)\right] \mathrm{d} t^{\prime} .
\end{aligned}
$$

Furthermore, we can apply the method of images to incorporate an absorbing boundary, i.e. the leverage ratio upon default, along the $x_{1}$-axis with a drifted dynamics of the form $x_{1}^{*}(t)=-c_{2}(t)-4 \beta c_{1}(t)$ into our model, where the parameter $\beta$ is a real adjustable parameter controlling the movement of the leverage ratio upon default. The corresponding solution $\tilde{P}\left(x_{1}, t\right)$ is given by

$$
\tilde{P}\left(x_{1}, t\right)=\int_{-\infty}^{0} \mathrm{~d} x_{1}^{\prime} K\left(x_{1}, t ; x_{1}^{\prime}, 0\right) \tilde{P}\left(x_{1}^{\prime}, 0\right)
$$

where

$$
K\left(x_{1}, t ; x_{1}^{\prime}, 0\right)=G\left(x_{1}, t ; x_{1}^{\prime}, 0\right)-G\left(x_{1}, t ;-x_{1}^{\prime}, 0\right) \exp \left(-4 \beta x_{1}^{\prime}\right) .
$$


Then, in terms of the kernel $K\left(x_{1}, t ; x_{1}^{\prime}, 0\right)$, we can also derive the probability of default over a period of time $t$ as follows:

$$
P_{\text {def }}\left(x_{1}, t\right)=1-\int_{-\infty}^{0} \mathrm{~d} x_{1}^{\prime} K\left(x_{1} \exp \{\alpha(t)\}, t ; x_{1}^{\prime}, 0\right) .
$$

\section{References}

Auerbach, A. J. (1985). Real determinants of corporate leverages corporate capital structures in the United States. Chicago, IL: University of Chicago Press.

Black, F., \& Cox, J. (1976). Valuing corporate securities: Some effects of bond indenture provisions. Journal of Finance, $35,351-367$.

Black, F., \& Scholes, M. (1973). The pricing of options and corporate liability. Journal of Political Economics, 81, $637-654$.

Briys, E., \& de Varenne, F. (1997). Valuing risky fixed rate debt: An extension. Journal of Financial and Quantitative Analysis, 32, 230-248.

Collin-Dufresne, P., \& Goldstein, R. (2001). Do credit spreads reflect stationary leverage ratios? Journal of Finance, 56, $1929-1957$.

Delianedis G., \& Geske R. (1999). Credit risk and risk neutral default probabilities: Information about rating migrations and defaults, WorkingPaper, UCLA.

Duffie, D., \& Singleton, K. J. (1997). An econometric model of the term structure of interest-rate swap yields. Journal of Finance, 52, 1287-1322.

Fama E., \& French K. R., (1999). Testing tradeoff and pecking order predications about dividends and debt, Working Paper, University of Chicago.

Hui, C. H., Lo, C. F., \& Tsang, S. W. (2003). Pricing corporate bonds with dynamic default barriers. Journal of Risk, 5(3), $17-37$.

Jalivand, A., \& Harris, R. S. (1984). Corporate behavior in adjusting to capital structure and dividend targets: An econometric investigation. Journal of Finance, 39, 611-625.

Jarrow R., Lando A., \& Turnbull S. (1994). A Markov model for the term structure of credit spreads, Working Paper, Cornell University.

Jarrow, R., \& Turnbull, S. (1995). Pricing options on financial securities subject to default risk. Journal of Finance, 50, $53-86$

Johnson, R. E. (1965). Term structures of corporate bond yields as a function of risk of default. Journal of Finance, 22 , $313-345$.

Lo, C. F., Lee, H. C., \& Hui, C. H. (2003). A simple approach for pricing Black-Scholes barrier options with timedependent parameters. Quantitative Finance, 3, 98-107.

Longstaff, F., \& Schwartz, E. (1995). A simple approach to valuing risky fixed and floating rate debt. Journal of Finance, $50,789-819$.

Madan D., \& Unal H., (1993). Pricing the risks of default, Working Paper, University of Maryland.

Marsh, P. (1982). The choice between equity and debt: An empirical study. Journal of Finance, 37, $121-144$.

Merton, R. (1974). On the pricing of corporate debt: The risk structure of interest rates. Journal of Finance, 29, 449-470.

Opler T., \& Titman S., (1995). The debt-equity choice, Working Paper, Ohio State University and Boston College.

Shyam-Sunder, L., \& Myers, S. C. (1999). Testing static tradeoff against pecking order models of capital structure. Journal of Financial Economics, 51, 219-244.

Standard \& Poor's. (2001). Adjusted Key U.S. Industrial Financial Ratios.

Standard \& Poor's. (2002). Ratings Performance 2001.

Vasicek, O. A. (1977). An equilibrium characterisation of the term structure. Journal of Financial Economics, 5, $177-188$. 